\title{
Acciones educativas para afrontar algunos desafíos de la filosofía en el siglo XXI
}

\author{
Educational actions to face some challenges of Philosophy \\ in the XXI Century*
}

SONIA PARÍS ALBERT**

\begin{abstract}
Resumen: Estas reflexiones proponen algunas acciones educativas para recuperar el valor de la filosofía y con las que se cuestiona la noción de «utilidad» tan basada en criterios economicistas actualmente. Para ello, se rescata en la educación formal, a la pedagogía libertaria de Freire desde edades tempranas, aprovechando la metodología de la filosofía para niños. Esta práctica pedagógica es una herramienta que visibiliza la utilidad de la filosofía para todas las personas y que fomenta la creatividad para abordar algunos problemas filosóficos. Así, se afrontan algunos de los que se entienden como desafíos de la filosofía en el siglo XXI.

Palabras clave: Desafíos de la filosofía; siglo XXI; pedagogía libertaria; filosofía para niños; pensamiento creativo.
\end{abstract}

\begin{abstract}
These thoughts propose some educational actions to recover the value of Philosophy, which help us to question the notion of «utility» so based on the economics criteria currently. For that, the libertarian education from early ages is rescued in the formal education, taking advantage of the Philosophy for Children methodology. This pedagogical practice is a tool that makes visible the utility of Philosophy for everyone and which promotes the creativity to approach some philosophical problems. Thus, some of the challenges understood as the ones of Philosophy in the XXI Century are faced.

Keywords: Challenges of Philosophy; XXI Century; libertarian education; Philosophy for Children; creative thinking.
\end{abstract}

Recibido: 11/09/2016. Aceptado: 20/11/2017.

* Este trabajo se enmarca en el proyecto de investigación «Testimonio ético y comunicación para el cambio: análisis de modos de re-significación de la figura de la víctima y de re-situación de los agentes sociales», con código P1·1B2015-21, financiado por la Universitat Jaume I de Castellón, España.

** Profesora Contratada Doctora del Departamento de Filosofía y Sociología de la Universitat Jaume I de Castellón, España. Principales líneas de investigación: Historia de la Filosofía, Filosofía para la Paz y Transformación Pacífica de Conflictos, Creatividad y Filosofía con Niñas y Niños. Publicaciones recientes: PARís Albert, Sonia (2016): «Indignación para la acción: un reencuentro con la filosofía de Friedrich Nietzsche», en Benet, Vicente J. y Álex Arévalo SAlinas (eds.): De víctimas a indignados. Imaginarios del sufrimiento y de la acción política, Valencia, Tirant Humanidades, pp. 205-218, España, ISBN: 978-8416556-45-8. - PARís Albert, Sonia (2017): «Filosofía para hacer las paces con niñas y niños. Un estímulo para la creatividad», Convergencia. Revista de Ciencias Sociales, México, agosto, núm. 75, pp. 65-85. ISSN: 1405-1435. Contacto: sparis@uji.es 


\section{Introducción}

La imagen que hoy impera de la filosofía es la de una reflexión abstracta, alejada de la cotidianidad de las personas. Distanciándose de esta interpretación, este trabajo propone el rescate de su utilidad como un desafío de la filosofía en el siglo XXI, junto con el reto de convertir a la reflexión filosófica en una actividad para todos y todas, capaz de abordar tanto los conocidos grandes problemas filosóficos del pensamiento occidental como otras cuestiones de interés para la humanidad según cada época.

Para afrontar los desafíos de la filosofía en el siglo XXI que en estas páginas se presentan, se plantea la práctica de otros modelos pedagógicos en la educación formal, entre los que se escoge la pedagogía libertaria de Freire. Concretamente, la proposición que se hace es la de incluir la pedagogía libertaria desde edades tempranas, siguiendo los principios expuestos por la escuela de filosofía para niños con Lipman. Finalmente, y subrayando que la formación filosófica desde los más pequeños y pequeñas favorece, entre otras capacidades, su aprendizaje en los pensamientos crítico, ético y creativo, se enfatiza este último como una herramienta, absolutamente necesaria, para encarar los desafíos de la filosofía aquí tratados, así como para la formación de una ciudadanía creativa y hábil en la búsqueda de soluciones alternativas a los problemas sociales.

\section{El rescate de la utilidad de la filosofía: una actividad para todos y para todas}

Cuando pensamos en la filosofía, y escuchamos hablar sobre ella, todos y todas recordamos, mayoritariamente, a los grandes filósofos de la historia del pensamiento occidental; ésos a quienes, continuamente en Occidente, hemos estudiado en los institutos y universidades, aunque, a decir verdad y en muchísimas ocasiones para gran parte del alumnado, sin entender demasiado bien el significado de sus reflexiones, el porqué de sus pensares ni su utilidad (Garcés, 2015). Por citar algunos casos, diré que me refiero aquí a filósofos como Sócrates, Platón, Aristóteles, San Agustín de Hipona, Descartes, Kant, Hegel y Nietzsche, entre otros. Todo un puñado de hombres filósofos ${ }^{l}$, cuyos nombres han destacado por encima del resto, y con el pasar del tiempo, gracias a la magnífica elaboración que hicieron de determinados sistemas teóricos, cuya finalidad fue la de responder a un buen conjunto de problemas filosóficos. Hombres filósofos que, siguiendo la terminología kantiana (Kant, 1978), se han enmarcado en lo que este autor denomina como la dimensión académica o escolar de la filosofía, según la cual se hace imprescindible buscar la unidad del conocimiento en un sistema. Hombres filósofos que, por lo tanto y de acuerdo con esta dimensión académica de la filosofía, pretendieron diseñar teorías filosóficas para dar sentido al conocimiento, abordando, así, algunos problemas filosóficos.

Esta noción de problemas filosóficos es la que, en este trabajo, nos permite avanzar un poco más en la comprensión de la dimensión académica de la filosofía (Kant, 1978). Para

1 Se utiliza el sustantivo hombre en masculino debido a que en la historia del pensamiento occidental ha sido a los hombres a quienes se ha destacado principalmente, constituyendo sus pensamientos la prácticamente totalidad de los currículums de las asignaturas de Historia de la filosofía de la educación secundaria y universitaria. Ésta ha sido la tendencia generalizada hasta bien entrada la contemporaneidad, cuando las mujeres han empezado a estar mucho más reconocidas en el ámbito de la reflexión filosófica. 
este fin, resulta interesante recuperar su definición etimológica, según la cual el significado de la palabra filosofía es «amor por la sabiduría», lo que viene a suponer el deseo constante por querer saber más sobre las cosas; por no querer quedarse en las puras apariencias (Corominas, 1961, 267) ${ }^{2}$. En este sentido, la filosofía está fuertemente unida a la capacidad de hacernos preguntas sobre todo aquello que nos envuelve, así como de pensar, reflexionar y discutir sobre esas mismas preguntas (Pieper, 1970).

Fruto de esa disposición curiosa a plantearse preguntas, a cuestionarlo todo y a pensar y discutir sobre esos interrogantes, han visto la luz lo que hemos conocido en Occidente como los grandes problemas filosóficos. Ni que decir tiene que la dimensión académica de la filosofía se ha referido, especialmente, a estos grandes problemas, los cuales se han mantenido con el tiempo, limitándose, en términos generales y como afirma Montero (1978, 19), «a renovaciones bizantinas del vocabulario empleado», causando, de esta manera, que la reflexión filosófica haya consistido, en muchas ocasiones, «en circunloquios sobre esos mismos problemas, a sabiendas de que son insolubles y que podían ahorrarse el esfuerzo de renovar las anteriores teorías» (Montero, 1978, 19).

Esa concepción de la filosofía como actividad que no deja avanzar en la creación del conocimiento, que se limita a debatir y dialogar siempre sobre los mismos problemas sin aportar ningún tipo de solución, es la que ha puesto en tela de juicio su utilidad (Garcés, 2015). Es necesario señalar aquí, que las críticas hacia la utilidad de la filosofía surgen al tomar como paradigma la interpretación que hoy en día se da al término de lo útil, siendo éste último identificado como prácticamente sinónimo de los adjetivos «productivo» y «rentable». De productivo si se tiene en cuenta que útil será el estudio de aquellas ciencias que sirven para algo porque llevan a la producción tangible de algo, en un mundo en el que el valor de todo es medido con criterios económicos y en función del precio que tienen en el mercado. De rentable si se toma en consideración que útil será, también, el estudio de aquellas ciencias que son rentables al sistema al revertirle en positivo, gracias a la manera en la que se identifican con los criterios economicistas de la utilidad. Así, tanto el calificativo de «productivo» como el de «rentable» llevan, irremediablemente, hacia la idea del rendimiento (Garcés, 2015), pues útil será, también, el estudio de las ciencias que favorecen el rendimiento para la producción de nuevas cosas en «un mundo totalitario del trabajo» (Pieper, 1970, 23); un mundo en el que, ciertamente, el trabajo ocupa toda nuestra energía, generando una gran presión por el rendimiento (Han, 2012,29), así como una gran crisis temporal marcada por la falta de una armonía en el tiempo que de un sentido a la vida (Han, 2015). Con todo ello se ha ido originando, en terminología de Han (2012), una sociedad del cansancio en la que hay una desvalorización del ser y una pérdida del ser humano en el mundo, quien acaba, finalmente, identificándose como un simple trabajador (Han, 2012). De esta manera, se ha construido «un mundo extraordinariamente inestable» (Vargas Lozano, 2010, 50), en el que el futuro «recupera su naturaleza de absoluta contingencia» (Bodei, 2008, 11) y donde las personas han acabado viviendo cargadas de inseguridades (Beck, 2006), motivadas, de manera prácticamente exclusiva, por el rendimiento logrado en sus trabajos a través de su capacidad productiva y rentable, y sin prestar casi atención a los pocos momentos que se

2 Esta definición se puede complementar con aquella otra que parte del concepto de thaumatzein, el cual implica una actitud de extrañeza y admiración (Martínez Guzmán, 2001). 
tienen para la reflexión y el pensar; falta de atención que se debe muchas veces a que no se puede pensar, al no disponer de casi tiempo para ello (Han, 2012; 2015), y otras muchas a que no se quiere, simplemente por no haber puesto en práctica habitualmente esta capacidad reflexiva (Freire, 2015).

La noción social de la utilidad, a la que me estoy refiriendo en estas páginas, es la que, también, se reproduce, actualmente, en la gran mayoría de centros educativos, muy especialmente en las universidades, donde encontramos que:

[...] todo profesor e investigador, sea del ramo que sea, tiene que ser única y exclusivamente un productor de artículos de investigación científica de impacto en los rankings internacionales de evaluación de la investigación (Garcés, 2015, 66).

Así, en los centros educativos, por un lado y sobre todo en el ámbito universitario, la utilidad va ligada a la redacción de determinados papers, no de todos, sino de aquellos que podrán ser publicados en las revistas científicas de gran impacto, dejando en un segundo lugar las otras tareas propias del profesorado, incluso aquellas que se relacionan directamente con la docencia. Por otro lado, y en todos los niveles educativos, ya no sólo en el universitario, la utilidad está vinculada, también, con el modo de proceder propio del modelo pedagógico bancario, el cual, habiendo sido desmenuzado hace ya bastantes años por Freire $(1970 ; 1993 ; 2001)$, sigue ocupando una posición prioritaria en la mayoría de los centros educativos. Se hace importante señalar en estas páginas, aunque sea a modo de síntesis recordatoria, que la utilidad para el modelo pedagógico bancario está unida a la capacidad de un estudiantado pasivo de reproducir, cuanto más al pie de la letra sea mejor, los mensajes transmitidos, a través de monólogos teatrales (Bourdieu y Passeron, 2001, 132), por el profesorado, quien, convertido en una autoridad estatuaria (Bourdieu y Passeron, 2001, 131) y visto como un banco de conocimientos (Freire, 1970; 1993; 2001), tiene la potestad de decidir qué se tiene que hacer, cómo y qué es lo más importante (Bourdieu y Passeron, 1967, 71).

Como se puede observar, los valores que priman hoy y de manera generalizada, tanto en el ámbito social como en el educativo, son valores economicistas, relacionados con un sentido de utilidad basado en la productividad, la rentabilidad y el rendimiento y que, además, muy poco espacio, dejan para la reflexión y el auge de la filosofía. No cabe ninguna duda de que la actividad filosófica poco puede esperar en sociedades y en centros educativos motivados, tan especialmente, por estos valores y dirigidos por esta visión de la utilidad.

La pregunta ahora es: ¿Cuáles son algunos de los desafíos de la filosofía en el siglo XXI? En primer lugar, hacer visible y comprensible que, justamente, la utilidad de la filosofía está en su aparente inutilidad (Ordine, 2013). Es decir, en esa capacidad de cuestionamiento que, como decíamos en párrafos anteriores, la caracteriza fruto de su asombro ante el mundo. En esta línea Pieper $(1970,45)$ afirma: «Sí, es cierto: el filosofar no sólo no sirve de hecho para nada, sino que además no puede ni debe servir absolutamente para nada», y más aún si se tiene en cuenta que el filosofar «es más bien un hacer que tiene sentido en sí mismo, que no se legitima precisamente por su aptitud para «servir para un fin» (Pieper, 1970, 48). Percatarnos de ello, de que la filosofía no «debe servir para ningún fin último», según señala 
Pieper (1970), y de que su utilidad está en su fuerza pensante y reflexiva, favorece su revalorización, al tiempo que esta revalorización hace posible una transvaloración basada en el hecho de ya no enfatizar los criterios economicistas y de utilidad antes mencionados, sino muy principalmente, la valía del tiempo para que las personas se asombren ante el mundo, cuestionándose las cosas, pensándolas, reflexionándolas y estableciendo debates y diálogos sobre ellas (Garcés, 2015). En estas últimas actitudes es donde está otro de los sentidos de lo que realmente será útil una vez lograda esta transvaloración, causada por la reivindicación de la aparente inutilidad de la filosofía (Ordine, 2013).

En segundo lugar, concebir la filosofía como una actividad para todos y para todas gracias al rescate de su utilidad. En cierta manera, esta es una idea que muchos otros libros han querido ya proponer, superando, también así, aquella otra visión que trataba de vincularla, exclusivamente, con unos pocos hombres filósofos. Y es que es natural a todo ser humano preguntarse por las cosas y buscar explicaciones (Terricabras, 1999); es natural a todo ser humano el hecho de filosofar, siendo esta última «una actitud humana fundamental frente al mundo, actitud que en gran manera es ajena a toda posición y disposición elegidas a voluntad» (Pieper, 1970, 25). En este sentido, la filosofía pasa a ser «una forma de compromiso con el mundo», que más «allá de los compromisos particulares, hace suyo el compromiso de hacer y de tener el mundo (Garcés, 2015, 15).

Esta aproximación de la filosofía a todos y a todas permite recuperar, al mismo tiempo, la dimensión mundana de la filosofía de la que también hablaba Kant (1978), en comparación con la dimensión académica mencionada, previamente, en estas páginas. La dimensión mundana de la filosofía es aquella que engloba todas las posibles preguntas que son parte de la razón humana, que todo ser humano puede plantearse, tanto en lo que a los grandes problemas filosóficos concierne como a nuevas cuestiones que resultan interesantes abordar según los intereses, preocupaciones y necesidades de cada época. Así, la dimensión mundana de la filosofía permite incorporar nuevos temas de interés para todos los seres humanos (Garcés, 2015), como pueden ser, actualmente, las cuestiones relativas al género, al terrorismo, a la regulación de conflictos por medios pacíficos, etc. Por supuesto que con esta dimensión se logra ampliar las fronteras de la reflexión filosófica, haciéndola, en muchas ocasiones, mucho más cotidiana y cercana a la gente. En esta línea de pensamiento, señala Martínez Guzmán (2001, 20): «Nos urge sacar la filosofía a la calle, al mundo distanciado del reconocimiento de los seres humanos como seres humanos y confrontarlo con las propuestas de los filósofos», pues no debemos olvidar que la «filosofía nació al aire libre y a las calles» (Garcés, 2015, 10) debe volver. La filosofía debe llevarse a la sociedad (Vargas Lozano, 2012) a través de un lenguaje sencillo y transparente, que facilite la comprensión de los mensajes, despojándose, en lo posible, del «simbolismo [...] de las lenguas históricas», es decir, de un lenguaje caracterizado por signos y por una terminología artificial (Pieper, 1970, 118).

En tercer y último lugar, incluir el reto de encontrar el equilibrio entre la dimensión académica y mundana de la filosofía, confrontando, como señalaba Martínez Guzmán $(2001,20)$ en su cita anterior, las propuestas de una dimensión con las de la otra para seguir reivindicando la utilidad de la filosofía, y con ello, poder poner el énfasis, también, en la visión que de ella se tenía como una forma de vida en la Antigua Grecia (Garcés, 2015, 9; Hadot, 2009, 67). 


\section{La pedagogía libertaria como práctica para encarar algunos desafíos de la filosofía en el siglo XXI}

En el apartado anterior se ha hecho hincapié en tres desafíos de la filosofía en el siglo XXI. Desafíos respecto a los que ahora se quiere destacar que su posibilidad de ser afrontados pasa, especialmente, por la práctica en las aulas de otros modelos pedagógicos ${ }^{3}$, los cuales, alejados de la forma de proceder de la pedagogía bancaria que critica Freire (1970; 1993; 2001), incitan mucho más al pensamiento, a la reflexión, al debate y al diálogo con una mayor presencia, entonces también, de la filosofía (Vargas Lozano, 2012), pues no hay duda de que el papel de la filosofía es fundamental si se considera que:

Lo que la filosofía como práctica educativa plantea es que educar no es adquirir competencias, transmitir conocimientos ni escolarizar pensamientos. Consiste, fundamentalmente, en un desplazamiento, en un cambio de lugar que renueva el deseo de pensar [...] (Garcés, 2015, 75).

Deseo de pensar que surge teniendo en cuenta que:

La filosofía no posee recetas, pero nos puede ayudar a presentar los ingredientes que forman parte de la elaboración de los juicios y, en la medida en que puede hacer esto con éxito, no puede dejar de afectar nuestro carácter para bien (Lipman, 1998, 122).

Y actuar de acuerdo con los contenidos de estas dos citas, requiere poner encima de la mesa, también, que el «maestro, en filosofía, no forma ni adiestra, libera: libera de lo que nos impide pensar. El verdadero maestro es, en última instancia, el maestro que nos libera de la tutela del maestro» (Garcés, 2015, 76).

Entonces, tomando como punto de partida de las distintas prácticas pedagógicas que se quieren promover, las ideas planteadas en las citas anteriores, se escoge, a modo de referencia para poder afrontar los tres desafíos expuestos en la sección anterior, la propuesta alternativa al modelo pedagógico bancario que el mismo Freire elabora. Esto es, el modelo problematizador (Freire, 1970; 1993; 2001) o modelo libertario (Freire, 2015). A modo de anotación, cabe decir aquí que se escoge este modelo freiriano, aun teniendo en cuenta que ha recibido ciertas críticas, como, por ejemplo, las que vienen de la mano de Kusch (2000), para quien el enfoque freiriano de promover el desarrollo a través de la educación es falible en sí mismo, pues, según este autor, Freire propone un modelo educativo occidental que trata de aplicar, con un sentido general, a cualquier pueblo o cultura y olvida, con ello, el carácter local de toda educación, es decir, su sentido de acuerdo con y para cada comunidad. Una crítica que Kusch (2000) elabora como resultado del valor que da al aspecto interno en su visión del desarrollo frente al externo y con el que sitúa a lo local en una posición prioritaria en la educación frente a las influencias externas.

3 En estas páginas se hace hincapié en el papel de la educación formal aún a sabiendas de que para encarar los tres desafíos expuestos en el primer apartado se requiere, también, la colaboración de la educación no formal e informal. En este sentido, García Moriyón (2004) defiende, por ejemplo, la necesidad de una estrecha vinculación entre la familia y la escuela. 
Sin olvidar estas críticas, ciertamente, lo que se pretende destacar en este texto, de acuerdo con las ideas que se viene trabajando, es que la pedagogía libertaria incita mucho más a la reflexión, siendo proclive al desarrollo de la capacidad de pensamiento y cuestionamiento, gracias a la subversión de roles que impulsa, según la cual tanto el colectivo del profesorado como el del estudiantado educa y es educado, desempeñando, así, el estudiantado un papel mucho más activo, al reconocer que debe opinar sobre su propio proceso de aprendizaje, haciendo oír sus voces, valorando los contenidos a trabajar, complementando, con sus experiencias de vida y saberes, las perspectivas con las que dichos contenidos se analizan, y asumiendo, por encima de todo, una postura propia. Es de esta manera que este estudiantado deja de estar alineado, deja de asumir su propia ignorancia, se reconoce como un sujeto importante, comprometido con el proceso de aprendizaje-enseñanza (Freire, 2015, 152), y se percibe, al mismo tiempo, como un sujeto explorador, motivado por la sorpresa, por lo imprevisible, y con gran capacidad moral y empática (Greene, 2005).

Tiene lugar, entonces, en el marco de esta pedagogía, un mayor equilibrio en la relación entre el alumnado y el profesorado, ya que ambos dejan de estar encerrados en las posiciones en las que tradicionalmente se han encontrado (el profesorado en la de educador y el alumnado en la de educado), para abrirse a nuevos escenarios en los que se establece un mayor reconocimiento entre ambas partes, más aún, si tenemos en cuenta que cada una de ellas tiene elementos que aportar en el proceso de aprendizaje. Es decir, para la pedagogía libertaria, el aprendizaje ya no es fruto sólo de los conocimientos transmitidos por el profesorado, sino también de aquellos otros que el alumnado puede proporcionar, teniendo estos últimos (los del estudiantado), exactamente, la misma valía que los primeros (los del profesorado) (Freire, 1970; 1993; 2001). De esta manera, es como el aula se convierte, por un lado, en un centro de debate y de reflexión (Freire, 2015, 156), en el que el diálogo, como método de trabajo, es continuo (Freire, 2009) para poder escuchar las distintas voces, cuestionando todo aquello que se dice, argumentando las posiciones de cada cual, buscando las posibles contradicciones en los argumentos, así como los razonamientos afines, y permitiendo la introducción de nuevos temas relacionados con el currículo general de la asignatura en cuestión, aún a expensas de que algunas de sus unidades didácticas no vayan a poder ser trabajadas. Por otro lado, el aula se transforma en un espacio plural, como la vida misma según Perrenoud (2007), en la que se parte del presupuesto de que ningún estudiante es igual a otro y, por consiguiente, de que todas las personas dentro del aula deben ser reconocidas en su individualidad, teniendo en cuenta sus similitudes y diferencias.

Este reconocimiento plural al alumnado y al papel que puede desempeñar en el proceso de aprendizaje-enseñanza de acuerdo con la pedagogía libertaria, construye un estudiante liberado y emancipado, quien, según Marina (2011), se encuentra más motivado y con estilos afectivos que favorecen el bienestar y la convivencia (Marina, 2010, 87), dando lugar, así, a una pedagogía del entusiasmo capaz de contagiar las ganas de aprender y de saber. No cabe ninguna duda, pues, de que la filosofía tiene mucha más cabida en el marco de esta pedagogía del entusiasmo fruto de la pedagogía libertaria por sus propias características, que son inherentes, al mismo tiempo, a la filosofía. Es decir, esta práctica educativa, basada en el modelo pedagógico libertario, incita mucho más al pensamiento, a la reflexión, al debate y al diálogo, con lo que la filosofía puede tener una mayor presencia en ella, permitiendo, de esta manera, seguir trabajando para encarar los tres desafíos expuestos en el primer apartado de este trabajo. Tanto 
es así que en lo que al primer desafío afecta, hacer visible y comprensible que, justamente, la utilidad de la filosofía está en su aparente inutilidad (Ordine, 2013), se puede añadir ahora, a lo dicho ya previamente, que la pedagogía libertaria facilita, por ejemplo, comprender que:

[...] la investigación filosófica puede ayudarnos a analizar las situaciones problemáticas en las que nos encontramos para separar lo que merece una consideración de lo que no la merece, lo que es pertinente de lo que no lo es, lo que es significativo de lo que no lo es (Lipman, 1998, 122).

En lo que respecta al segundo desafío, concebir la filosofía como una actividad para todos y para todas gracias al rescate de su utilidad, se puede completar, también lo ya dicho con anterioridad, indicando, por ejemplo, que esta práctica educativa ofrece la posibilidad de acercar la reflexión filosófica a los más pequeños y pequeñas, promoviendo el razonamiento, el pensamiento, el debate y la reflexión sobre los grandes problemas filosóficos, u otras cuestiones de interés filosófico propias de cada época, desde edades tempranas, facilitando, así y al mismo tiempo, el equilibrio entre la dimensión académica y mundana de la filosofía (Martínez Guzmán, 2001), tercer desafío también expuesto más arriba.

\subsection{Pedagogía libertaria desde edades tempranas: la escuela de filosofía para niños}

En la sección anterior se ha hecho un acercamiento a la pedagogía libertaria como modelo para afrontar los tres desafíos de la filosofía en el siglo XXI que se han expuesto desde el principio de estas páginas. En este apartado, lo que se quiere es enfatizar que el impulso de esta pedagogía no se debe vincular sólo con la educación secundaria y universitaria, sino también con la infantil y primaria, y así, plantear la pregunta de por qué no incorporarla desde los primeros niveles de la educación formal (Vargas Lozano, 2012) si, como se señalaba anteriormente, su práctica lleva aparejada la posibilidad de una mayor presencia de la filosofía. No se puede obviar que, mayoritariamente, la poca filosofía que se ha impartido, y que se está impartiendo hoy en día, es en los centros de educación secundaria y universitaria. Sin embargo, en este trabajo, se quiere poner el énfasis, sobre todo, en su inclusión en los colegios de educación infantil y primaria, a través de esta pedagogía libertaria, la cual, al ser mucho más reflexiva, puede habituar a los y las más pequeñas a pensar, formando, así y al mismo tiempo, personas que en un futuro puedan llegar a ser ciudadanas mucho más comprometidas (De la Garza, 1998; Sátiro, 2009a; 2011a).

Este apartado centrará, entonces, su atención en la incorporación de la filosofía en los primeros niveles de la educación formal, justamente, por la escasa presencia que ha tenido y tiene en estos niveles. Este énfasis en la inclusión de la reflexión filosófica en la educación infantil y primaria se puede entender, además, como una acción que, en el marco de la pedagogía libertaria, permite seguir trabajando para afrontar los tres desafíos expuestos en las primeras páginas de este texto. Anteriormente, ya se ha señalado cómo esta incorporación favorece la interpretación de la filosofía como una actividad para todos y todas. Así mismo, no hace falta decir que el hecho de aprender a filosofar desde los y las más pequeñas, tratando cuestiones varias de interés filosófico, beneficia tanto el rescate de su utilidad como el equilibrio entre las dos dimensiones (académica y mundana) ideadas por Kant (1978). 
La propuesta de integrar la filosofía en el currículo de los y las más pequeñas no es una idea novedosa, sino que se viene trabajando desde tiempo atrás, de la mano de distintos centros académicos, con la escuela de la filosofía para niños, cuyos principios se pueden poner en comunión con los de la pedagogía libertaria de Freire, en cuanto que la metodología de la escuela de filosofía para niños puede usarse para lograr un mayor carácter libertador y emancipador de los niños y las niñas, al estilo de lo que, también, Freire pretendió hacer, aunque, en su caso, con las personas adultas.

Mathew Lipman (1988a, 1988b) fue quien (tras percatarse de que algo estaba sucediendo con el estudiantado matriculado en sus clases de filosofía en la universidad, el cual no parecía mostrar demasiado interés en ellas ni siquiera comprenderlas), decidió crear el proyecto de la escuela de filosofía para niños con la finalidad de incorporar la reflexión filosófica en el currículo educativo desde los y las más pequeñas y para superar, al mismo tiempo, los problemas docentes con los que él se había encontrado (Hoyos Valdés, 2010). Su objetivo fue el de acercar la filosofía al alumnado, desde los y las más pequeñas, para que no les resultara extraña al llegar a la educación secundaria y a la universidad. No se puede dejar de mencionar que este proyecto lo hizo muy influenciado por Leonard Nelson, quien consideraba que era imprescindible «enseñar a filosofar a través de una didáctica no dogmática, que privilegiase el descubrimiento personal de los alumnos» (Arnaiz, 2007, 36). Así, a través de la lectura grupal de cuentos adaptados a la edad de los niños y las niñas, Lipman propone tratar en el aula cuestiones que afectan a la vida de las personas y sobre las que los niños y niñas pueden dialogar. Su propuesta de clases, entonces, no tiene ningún programa predefinido, sino que, más bien, consiste en una serie de diálogos establecidos entre los y las más pequeñas a partir de la lectura de un cuento, gracias al cual y de acuerdo con sus intereses y preocupaciones, los niños y las niñas pueden reflexionar (Haynes, 2004). De esta manera, el o la docente debe esforzarse por conseguir la participación de los y las más pequeñas, animando el debate mediante la introducción de herramientas conceptuales para generar ideas (París Albert, 2015, 212-213). Esta última es una cuestión nada fácil si se tiene en cuenta que para ejercer su rol adecuadamente, el profesorado debe ser un gran conocedor de la historia de la filosofía occidental. Así mismo, entre sus funciones está la de respetar el ritmo de los niños y las niñas, sin marcar objetivos predefinidos, ni la necesidad de alcanzar soluciones, aunque estableciendo de forma clara, y desde el principio, las reglas del juego del quehacer filosófico, las cuales, según Hoyos Valdés $(2010,154)$, son: tratar de ser relevante, consistente, coherente y no auto-contradictorio. A todo ello se añade su esfuerzo constante por mostrar los sentires ocultos detrás de las opiniones de los niños y las niñas, y las debilidades y fortalezas de sus argumentos, pidiendo evidencias de lo que los y las más pequeñas afirman y mostrando los efectos de sus opiniones (Hoyos Valdés, 2010, 160). Es evidente que estos son algunos de los motivos por los que la escuela de filosofía para niños requiere un cambio en los materiales y en la estructura de sus clases, pasando a entender las aulas como comunidades de indagación filosófica (Lipman, 1992; Sátiro, 2012), donde los niños y las niñas pueden revisar «sus planteamientos y los de los otros» (Hoyos Valdés, 2010, 157), y donde cada pequeño y pequeña puede ser vista como una nueva indagadora filosófica, capaz de ofrecer interpretaciones diferentes sobre un mismo tema. Se trata, pues, de hacer hincapié en el diálogo filosófico con el fin de aprender a aprender, de dar sentido al mundo y de afrontar los retos de una sociedad cada vez más complicada (García Moriyón, 2006). 
Cuando Lipman (1992) define las aulas como comunidades de indagación filosófica, tal y como se indica en el párrafo anterior, está entendiendo que la filosofía es accesible a todos los niños y niñas por tres motivos concretamente: En primer lugar, por la urgencia de reconocerlos como sujetos activos con quienes se debe poder compartir los problemas y los logros de la sociedad en la que viven (De la Garza, 1998, 133-134). Se trata, pues, de reconocer su voz en la esfera pública (Canelles y otros, 2016; García Moriyón y otros, 2012; Haynes, 2004, 35), como personas pensantes que pueden imaginar ideas creativas para la consecución de los retos sociales, dejando atrás la visión que de ellas se ha tenido, y se sigue teniendo, según la cual es mejor mantenerlas alejadas de los problemas. Es decir, ciertamente, como señala Kohan (2011), autor que hace una revisión crítica del modelo de Lipman cuando propone el paso desde la filosofía para niños a la filosofía con niños, se trata de subvertir su visión como la de infantes que están en una determinada etapa de la vida (Kohan, 2011, 341), en la que se preparan para la vida adulta (Storme y Vliegue, 2011, 184) para empezar a concebirlos como sujetos que se encuentran en un proceso de maduración (Murris, 1999), en el que deben ser reconocidos a través de sus particulares fortalezas, lo cual les habilita para disfrutar de una vida de calidad plena más allá de cualquier edad cronológica (Kohan, 2011, 342). En segundo lugar, Lipman percibe que la filosofía es accesible a los niños y las niñas por la propia función que cumple para el desarrollo integral del ser humano, tal y como ya se ha venido reseñando en estas páginas (De la Garza, 1998, 133-134). Por decirlo en otros términos que complementan lo ya expuesto anteriormente, se incluye la siguiente cita:

Si los alumnos aprenden a pensar críticamente, no estarán indefensos frente a los intentos de adoctrinamiento; si aprenden a escuchar cuidadosamente a los otros, tomando en consideración otras perspectivas, aprenderán a respetarlas [...] (De la Garza, 1998, 136).

A modo de inciso, cabe decir que, hasta el momento, este trabajo se ha referido a la noción de pensamiento en término generales. Sin embargo, en la cita anterior se delimita un poco más este concepto de acuerdo con Lipman, siendo, al mismo tiempo, ésta la visión que nos permite seguir avanzando en este estudio. Concretamente, en la cita se destacan dos tipos de pensamiento: el pensamiento crítico y ético. Cuando Lipman habla del primero se refiere a aquel pensamiento que se puede utilizar para dar sentido a los textos que leemos, las imágenes que vemos o las palabras que escuchamos con el fin tanto de poder opinar al respecto como de decidir si se está o no de acuerdo con ello (Pritchard, 1998, 74-75). Con la noción de pensamiento ético, en cambio, Lipman alude a la posibilidad de ponernos en la posición de otras personas, comprendiendo de manera empática los distintos puntos de vista sobre una misma cuestión (Greene, 2005).

A estos dos tipos de pensamiento, el crítico y el ético, hay que añadir, según este autor, un tercero, el pensamiento creativo, el cual, aunque no se menciona en la cita anterior, es igual de importante (Sátiro, 2002, 145). Justamente, es a una breve caracterización de este pensamiento creativo, por su papel en la escuela de filosofía para niños, al que se referirá el siguiente apartado. No obstante, se puede avanzar aquí que la formación filosófica desde edades tempranas, siguiendo los principios de la escuela de filosofía para niños, además de 
potenciar, por ejemplo, la capacidad reflexiva y para el diálogo de los niños y las niñas, favorece también su disposición para los pensamientos crítico, ético y creativo (De Puig y Sátiro, 2010; Sátiro, 2002). Todo ello en pro del sentido de utilidad, alejado de criterios economicistas, que se señalaba en la primera sección, así como de los tres desafíos de la filosofía en el siglo XXI trabajados en este texto.

En tercer y último lugar, Lipman (1992) ve la filosofía asequible para los y las más pequeñas a causa de la ineludible necesidad que se manifiesta en las sociedades actuales de aprender a filosofar, para que, cuando estos niños y niñas sean personas adultas, estén habituadas a poner en práctica su capacidad reflexiva, dialógica y del pensar (De la Garza, 1998, 133-134; Sátiro, 2009a; 2011a).

En definitiva, la comunidad de indagación filosófica «propone ejercitar la mente por medio del pensamiento disciplinario y estimulante y de una interacción estructurada» (Haynes, 2004, 33), en la que el alumnado aprende a objetar el razonamiento débil, construir argumentos sólidos, aceptar la responsabilidad de sus contribuciones, admitir su dependencia con respecto a los otros y las otras, practicar de forma colaborativa en la autocorrección, seguir la investigación hasta dónde ésta les lleve, sentirse personas orgullosas de los avances del grupo como un todo y de los suyos propios (De la Garza, 1998, 137), y desarrollar su pensamiento creativo.

\section{La relación inapelable entre el pensamiento creativo y algunos desafíos de la filoso- fía en el siglo XXI}

Decía la sección anterior que aprender a filosofar desde edades tempranas hace posible desarrollar los pensamientos crítico, ético y creativo, lo cual favorece el hecho de que en el futuro las personas puedan llegar a ser ciudadanas mucho más comprometidas (Sátiro, 2009a; 2011a). De estos tres pensamientos y como se indicaba en el apartado anterior, quisiera destacar el tercero, el pensamiento creativo, ya que se quiere enfatizar la imagen de la creatividad como la de una herramienta absolutamente necesaria para la búsqueda de soluciones alternativas a los problemas sociales. No cabe ninguna duda de que formar en la creatividad hace que los niños y las niñas, al llegar a edades adultas, sean mucho más capaces de afrontar las situaciones cotidianas de la vida con respuestas distintas a las acostumbradas. Además, este empeño en la creatividad, derivado del impulso en la formación filosófica, permite seguir trabajando en pro de los tres desafíos de la filosofía expuestos en estas páginas.

¿Qué es la creatividad? Para empezar, cabe decir que la noción de creatividad que aquí se propone es la misma que se trabaja en la escuela de filosofía para niños, muy especialmente, de la mano de Sátiro, autora que, en el marco de esta escuela, hace hincapié en el pensamiento creativo. Para ella, siguiendo las investigaciones de Csikszentmihaly (1998), la creatividad es la capacidad humana que requiere captar fuentes de la experiencia (Sátiro, 2002, 147) para generar más, nuevas y mejores ideas (Sátiro, 2011a, 136), estando totalmente vinculada con las habilidades de imaginar y razonar analógicamente (Sátiro, 2005, 47), y siendo la flexibilidad, la originalidad, la fluidez y la elaboración sus principales rasgos (Sátiro, 2005, 47). Además, señala los siguientes como los cuatro elementos de la creatividad (Sátiro, 2002; 2013): el producto creativo, que es la parte tangible y 
perceptible por los sentidos. La persona creativa, la cual se define especialmente por su complejidad $^{4}$. El ambiente creativo, el cual es muy importante porque puede estimular o no la creatividad. Por ejemplo, para la escuela de filosofía para niños, el aula entendida como una comunidad de indagación filosófica es un entorno idóneo para avivar el pensamiento creativo (Sátiro, 2002, 154). Y el proceso creativo, compuesto por distintas fases como son la de preparación, incubación, intuición, evaluación, elaboración y comunicación (Sátiro, 2002, 157).

Hay que indicar, también, que, al definir la creatividad como capacidad humana, Sátiro está mostrando su presencia en todas las personas (Rojas Chávez, 2015, 187), aunque deje bien claro que puede haber grados distintos en su desarrollo, y por este mismo motivo, personas que son mucho más creativas que otras (Sátiro, 2011b: 4). Sin embargo, para la autora «el hecho que existan genios no significa que los demás no sean creativos (Rojas Chávez, 2015, 187). Ella misma escribe:

La creatividad es una capacidad humana que hace que, de manera inusitada y original, el ser humano, contextualizado en su entorno, se amplíe y profundice individual y colectivamente en distintos ámbitos. No es algo exclusivo de personas especiales y genios, sino algo que puede ser desarrollado por cualquier persona (Sátiro, 2009c, 98).

En resumen, si la creatividad es una capacidad que se encuentra en todas las personas, lo que nos interesa es educar en ella a través de la práctica de nuevas pedagogías (Sátiro, 2011a), como la libertaria, y con una mayor presencia de la filosofía desde los y las más pequeñas, como promueve la escuela de filosofía para niños, incitando a una ciudadanía motivada por una forma de pensar, de ser y de actuar e interactuar creativa (Sátiro, 2011a, 133 ; 2013), capaz de ver la vida como una obra de arte y la humanidad como un proyecto (Sátiro, 2009b, 53). Estas son las actitudes que, derivadas de la formación filosófica, nos ayudarán a acercarnos, cada vez más, a un sentido de utilidad alejado de los criterios economicistas expuestos al principio de este texto, así como, también, a poder encarar los tres desafíos de la filosofía en el siglo XXI, dejando entrever su ineludible relación con la creatividad. Todo ello gracias a una revalorización de la reflexión filosófica desde los y las más pequeñas, fruto de la puesta en práctica de nuevas maneras de aprender y de enseñar.

\section{Conclusiones}

La sociedad del siglo XXI requiere afrontar los tres desafíos de la filosofía que se han expuesto desde las primeras páginas de este texto con la finalidad de subvertir la propia imagen de la filosofía, para empezar a concebirla como una actividad útil, al alcance de

4 Según Sátiro (2002, 152; 2009b) y de acuerdo con Csikszentmihalyi, las características de la persona creativa son: gran cantidad de energía física, pero también necesidad de silencio y reposo, dominio experto en muchas situaciones, aunque ingenuidad en otras, responsabilidad e irresponsabilidad, imaginación y fantasía desarrolladas, aunque con gran sentido de la realidad, extroversión e introversión, humildad y orgullo, androginia psicológica, con manifestaciones masculinas y femeninas (agresividad y protección, sensibilidad y rigidez, dominación y sumisión), gran pasión por el trabajo, y apertura y sensibilidad ante el mundo, lo que proporciona placer y dolor. 
todos y de todas desde edades tempranas, con la que es posible abordar tanto los llamados grandes problemas filosóficos del pensamiento occidental como cualquier otra cuestión de interés para la humanidad según cada época.

Esta subversión de la noción de la filosofía supone, al mismo tiempo, un gran esfuerzo para conseguir una transvaloración del sentido de «utilidad», a través de la que se pueda poner en tela de juicio la ineludible necesidad de relacionar aquello que es útil con lo que es rentable económicamente hablando. Nuestros tiempos necesitan aprender a priorizar otros valores que humanicen el mismo sentido de utilidad y, con ello, que nos humanicen, también, a las persones, a las ciencias, a la economía, a la política, etc. Se trata, pues, de restar peso a los valores económicos para sumarlo a aquellos otros que están mucho más vinculados con la capacidad del pensamiento crítico, ético y creativo. Así, en esta línea de pensamiento, una de las propuestas que se hace en estas páginas es, justamente, la de enfatizar la creatividad frente al emprendedorismo tan presente hoy en día. Emprendedorismo que, sobre todo, resalta la capacidad para innovar acciones como potenciales negocios que han de maximizar la cantidad de beneficios sostenibles. Frente a ello, de la mano de la pedagogía libertaria y de la escuela de filosofía para niños, este texto resalta la posibilidad de aprender a ser personas creativas a través de una nueva mirada de la niñez, con la que los y las más pequeñas sean concebidas más allá de lo estrictamente cronológico, como sujetos que tienen derecho a hacer oír su voz y a ejercer un papel activo y comprometido en el marco de una ciudadanía crítica, ética y creativa.

\section{Bibliografía}

Arnaiz, G. (2007), «Evolución de los talleres filosóficos: de la filosofía para niños a las nuevas prácticas filosóficas», Childhood \& Philosophy, vol. 3, n 5, pp. 35-57.

Beck, U (2006), La sociedad del riesgo: Hacia una nueva modernidad, Barcelona: Paidós. Bodei, R. (2008), «Pensar el futuro. Incertidumbre y complejidad», Daimon. Revista de filosofía, suplemento 2, pp. 11-18.

Bourdieu, P. y Passeron, J. C. (1967), Los estudiantes y la cultura, Barcelona: Labor.

Bourdieu, P. y Passeron, J. C. (2001), La reproducción. Elementos para una Teoría del Sistema de Enseñanza, Madrid: Edición Popular.

Canelles, J. y otros (2016), «Filosofía lúdica sobre el temps i els diners», Perspectiva escolar, $\mathrm{n}^{\mathrm{o}} 385$, pp. 55-61.

Corominas, J. (1961), Breve diccionario etimológico de la lengua castellana, Madrid: Gredos.

Csikszentmihalyi, M. (1998), Creatividad. El fluir y la psicología del descubrimiento y la invención, Barcelona: Paidós.

De la Garza, M. T. (1998), «La comunidad de investigación como medio de educación moral», en: F. García Moriyón (ed.): Crecimiento moral y filosofía para niños, Bilbao: Desclée de Brouwer, pp. 125-145.

De Puig, I. y Sátiro, A. (2010), «Jugar a pensar con niños y niñas: ¿Por qué? ¿Para qué? Y otras preguntas más...», Crearmundos, nº 8, pp. 6-12.

Freire, P. (1970), Pedagogía del oprimido, México: Siglo XXI. 
Freire, P. (1993), Pedagogía de la esperanza: un reencuentro con la pedagogía del oprimido, México: Siglo XXI.

Freire, P. (2001), Pedagogía de la indignación, Madrid: Morata.

Freire, P. (2009), La educación como práctica de la libertad, Madrid: Siglo XXI.

Freire, P. (2015), Pedagogía liberadora, Madrid: Los Libros de la Catarata.

Garcés, M. (2015), Filosofía inacabada, Barcelona: Galaxia Gutemberg.

García Moriyón, F. (2004), Familia y escuela, Madrid: CCS.

García Moriyón, F. (2006), Pregunto, dialogo, aprendo: cómo hacer filosofía en el aula, Madrid: Ediciones de La Torre.

García Moriyón, F. y otros (2012), «¿Qué hay de filosófico en filosofía para niños?», Crearmundos, $\mathrm{n}^{\circ} 10$, pp. 5-12.

Greene, M. (2005), Liberar la imaginación: ensayos sobre educación, arte y cambio social, Barcelona: Graó.

Hadot, P. (2009), La filosofía como forma de vida. Conversaciones con Jeannie Carlier y Arnold I. Davidson, Barcelona: Alpha Decay.

Han, B. Ch. (2012), La sociedad del cansancio, Barcelona: Herder.

Han, B. Ch. (2015), El aroma del tiempo: un ensayo filosófico sobre el arte de demorarse, Barcelona: Herder.

Haynes, J. (2004), Los niños como filósofos. El aprendizaje mediante la indagación y el diálogo en la escuela de primaria, Barcelona: Paidós Ibérica.

Hoyos Valdés, D. (2010), «Filosofía para niños y lo que significa una educación filosófica», Discusiones filosóficas, año 11, n 16, pp. 149-167.

Kant, I. (1978), Crítica a la razón pura, Madrid: Alfaguara.

Kohan, W. O (2011), «Childhood, Education and Philosophy: Notes on Deterritorialisation», Journal of Philosophy of Education, vol. 45, n 2, pp. 339-357.

Kusch, R. (2000), Obras completas, Tomo III, Buenos Aires: Editorial Fundación Ross.

Lipman, M. (1988a), Investigación ética, Madrid: Ediciones de la Torre.

Lipman, M. (1988b), Investigación filosófica, Madrid: Ediciones de la Torre.

Lipman, M. (1992), La filosofía en el aula, Madrid: Ediciones de la Torre.

Lipman, M. (1998), «El papel de las narraciones en la educación moral», en: F. García Moriyón (ed.): Crecimiento moral y filosofía para niños, Bilbao: Desclée de Brouwer, pp. 115-124.

Marina, J. A. (2010), La educación del talento, Barcelona: Planeta.

Marina, J. A. (2011), Los secretos de la motivación, Barcelona: Planeta.

Martínez Guzmán, V. (2001), Filosofía para hacer las paces, Barcelona: Icaria.

Montero, F. (1978), «La historicidad de la Filosofía», en: F. Montero y otros (eds.): La Filosofía Presocrática, Valencia: Departamento de Historia de la Filosofía de la Universidad de Valencia, pp. 13-47.

Murris, K. (1999), «Philosophy with preliterate children», Thinking: The Journal of Philosophy for Children, vol. 14, nº 4 , pp. 23-33.

Ordine, N. (2013), La utilidad de lo inútil, Barcelona: Acantilado.

París Albert, S. (2015), «Hacia una revalorización de la filosofía en diálogo con la indignación», Convergencia. Revista de Ciencias Sociales, nº. 68, pp. 207-229.

Perrenoud, Ph. (2007), Pedagogía diferenciada. De las intenciones a la acción, Madrid: Edición Popular. 
Pieper, J. (1970), Defensa de la filosofía, Barcelona: Herder.

Pritchard, M. (1998), «Desarrollo moral y filosofía para niños», en: F. García Moriyón (ed.): Crecimiento moral y filosofía para niños, Bilbao, Desclée de Brouwer, pp. 69-112.

Rojas Chávez, V. A. (2015), «Diálogo con Angélica Sátiro», Análisis, vol. 47, n 86, pp. 181.192.

Sátiro, A. (2002), «¿Crear? Un artículo para dialogar», en: M. Lipman (ed.): Filosofía y Educación, Madrid: Ediciones de la Torre, pp. 145-161.

Sátiro, A. (2005), «Cómo el pensamiento vuela cuando jugamos a pensar creativamente...», en: VVAA (coords.): Filosofía en la escuela. La práctica de pensar en las aulas, Barcelona: Graó, pp. 47-53.

Sátiro, A. (2009a), «Pasión por crear, placer de admirar, necesidad de transformar», en: A. Sátiro (coord.): Pasión por crear, placer de admirar, necesidad de transformar. Iniciación al arte como herramienta de juego, conocimiento y transformación, La Coruña: MACUF, pp. 8-13.

Sátiro, A. (2009b): «La propia vida como obra de arte: pasión por crearse a uno mismo», en: A. Sátiro (coord.): Pasión por crear, placer de admirar, necesidad de transformar. Iniciación al arte como herramienta de juego, conocimiento y transformación, La Coruña: MACUF, pp. 52-65.

Sátiro, A. (2009c), «Creatividad social: necesidad de transformar y crear mundos», en: A. Sátiro (coord.): Pasión por crear, placer de admirar, necesidad de transformar. Iniciación al arte como herramienta de juego, conocimiento y transformación, La Coruña: MACUF, pp. 96-115.

Sátiro, A. (2011a), «Pedagogia per a una ciutadania creativa», Temps d'Educació, n 40, pp. 129-144.

Sátiro, A. (2011b), «La creativitat com a motor de desenvolupament i d'inclusió social», Cantabou, no 33, pp. 4-13.

Sátiro, A. (2012), «La comunidad de investigación como cuna de la ciudadanía creativa», «QQué hay de filosófico en filosofía para niños», Crearmundos, n 10, pp. 5-12.

Sátiro, A. (2013), «La capacidad creativa como generadora del bien común», Crearmundos, $\mathrm{n}^{\mathrm{o}} 11$, pp. 51-62.

Storme, Th. y Vliegue, J. (2011), «The experience of Childhood and the Learning Society: Allowing the child to be philosophical and Philosophy to be Childish», Journal of Philosophy of Education, vol. 45, n 2, pp. 183-198.

Terricabras, J. M. (1999), Atrévete a pensar. La utilidad del pensamiento riguroso en la vida cotidiana, Barcelona: Paidós.

Vargas Lozano, G. (2010), «Los desafíos de la filosofía para el siglo XXI», Eikasia. Revista de filosofía, año 5, no 33, pp. 41-59.

Vargas Lozano, G. (2012), Filosofía ¿Para qué? Desafíos de la filosofía en el siglo XXI, México: Universidad Autónoma Metropolitana-Iztapalapa/Itaca. 
